Doug Geisler, Eva K. Grebel, and Dante Minniti, eds.

\title{
The Globular Cluster Systems of the Sculptor Group
}

\author{
Knut Olsen
}

Cerro Tololo Inter-American Observatory, Casilla 603, La Serena, Chile

Bryan Miller

Gemini Observatory, c/o AURA, Casilla 603, La Serena, Chile

Robert Schommer

U.S. Gemini Project Office, c/o AURA, Casilla 603, La Serena, Chile

Nick Suntzeff

Cerro Tololo Inter-American Observatory, Casilla 603, La Serena, Chile

John Bright

Harvard-Smithsonian Center for Astrophysics, 60 Garden St., Cambridge, $M A$ 02138, USA

\begin{abstract}
We present results from a study to identify the globular cluster systems of six of the brightest galaxies of the Sculptor Group. From Mosaic II $C M R$ images of the area surrounding the galaxies NGC 45, NGC 55, NGC 247, NGC 253, NGC 300, and NGC 7793, we identify cluster candidates through their morphology, luminosity, and color. We show that many of the Milky Way's globular clusters would appear extended in our images if placed in the Sculptor Group; only in the two most distant galaxies, NGC 45 and NGC 7793, is the discrimination difficult. We find 50-100 candidates per galaxy with integrated properties similar to Milky Way clusters. Published spectroscopic identifications of candidates in NGC 253 lead us to expect that $\sim 50 \%$ of the candidates are true clusters, while the remainder are background galaxies. Study of the Sculptor Group galaxies increases the sample of late-type spiral and Magellanic-type galaxies with known globular cluster systems from 2 to $\sim 10$.
\end{abstract}

\section{Introduction}

Discovering which processes dominate the formation of $\mathrm{GC}$ systems requires measurements of metallicities, ages, and kinematics of cluster systems in galaxies spanning the entire Hubble sequence. This conference has seen many exciting results from the study of GC systems surrounding elliptical galaxies. Since lowmass galaxies may feed the cluster systems of the larger galaxies, they are also 
important systems for study. Studies of a few low-mass galaxies have produced some surprises: 1) Miller et al. (1998) found similar specific frequencies of GCs in nucleated $\mathrm{dE}$ and giant $\mathrm{E}$ galaxies, showing that the efficiency of GC formation in dwarf galaxies can be as high as in the giants. 2) The red GCs in the LMC are as old as the Milky Way's oldest clusters to within 1 Gyr (Mighell et al. 1996, Olsen et al. 1998, Johnson et al. 1999), and are metal-poor (Olszewski et al. 1991). Their kinematics follow that of the HI disk (Schommer et al. 1992), implying that the disks of some galaxies were already in place by the time the MW halo formed. 3) M33's GCs reside both in a disk and in a halo (Chandar 2001, Schommer et al. 1991), yet the bulk have red horizontal branches, suggesting intermediate age (Sarajedini et al. 1998). Thus, halo kinematics need not be synonymous with old age.

\section{Why study the Sculptor Group's globular clusters?}

The Sculptor Group contains seven galaxies with luminosities similar to that of the LMC and M33, as well as 8 fainter dwarfs. Identifying their GC systems will increase the sample of late-type spiral and Magellanic-type galaxies with known systems to $\sim 10$, a meaningful size. The Sculptor Group is more loosely bound than the Local Group and contains no giant galaxies, allowing the study of GC systems in an environment less prone to violent interactions. The galaxies are nearby enough that the clusters will be resolved into stars with $10-\mathrm{m}+\mathrm{AO}-$ corrected ground-based telescopes.

Identifying globular clusters in the late-type Sculptor Group galaxies is challenging. The expected surface density of clusters is considerably lower than that of background galaxies and foreground stars, and the clusters are distributed over wide fields. Searches in NGC 55 and NGC 253, using photographic plates and spectroscopic follow-up, have turned up a handful of GCs (Da Costa \& Graham 1982, Liller \& Alcaino 1983a\&b, Blecha 1986, Beasley \& Sharples 2000). As shown by e.g. Blecha (1986), many of the clusters are resolved from the ground, which reduces foreground star contamination in lists of candidates. Contamination from background galaxies remains high, however; spectroscopic confirmation has revealed that only $15 \%$ of the photographically identified candidates are true clusters (Beasley \& Sharples 2000).

\section{Observations}

We have surveyed the six Sculptor Group galaxies NGC 45, NGC 55, NGC 247, NGC 253, NGC 300, and NGC 7793 with the CTIO 4-m telescope, Mosaic II camera, and Washington $C M$ and Harris $R$ filters. The $36^{\prime} \times 36^{\prime}$ field of Mosaic II captures $>50 \%$ of the expected area spanned by the GC systems in a single snapshot. The deep CCD images, which were taken under $\lesssim 1^{\prime \prime}$ seeing conditions, are superior to photographic plates for discriminating foreground stars and background galaxies from slightly broadened clusters. The Washington filter set provides the best metallicity sensitivity possible with broad-band filters, as well as additional star-galaxy discriminating power.

Fig. 1 demonstrates the ability of our images to discriminate Sculptor Group clusters from background galaxies. We estimate that with our data, remaining 
contamination from background galaxies and foreground stars will be $\$ 50 \%$. Follow-up spectroscopy is still required to confirm cluster candidates.

\section{Results}

From our $C M R$ photometry of the integrated light of known clusters in NGC 55 and NGC 253, we derive abundance estimates. Most of the clusters appear metal-poor, while one cluster, located in the halo of NGC 253 at a projected distance $\sim 10 \mathrm{kpc}$ from the galactic center, has solar metallicity.

We have used measurements performed with the program SExtractor (Bertin \& Arnouts 1996) to automatically identify new candidate Sculptor Group GCs. Our measurements include the magnitude within the isophote $3 \sigma$ above the surface brightness limit, the area contained within the isophote, and the ellipticity of the isophote. Fig. 2 illustrates how the Milky Way's GCs would appear in our main diagnostic diagram, which plots the isophotal area against flux. The clusters generally appear significantly broader than the stellar PSF, but are rounder and narrower than the majority of background galaxies.

We find that 50-100 objects each in NGC 45, NGC 55, NGC 247, NGC 253, NGC 300, and NGC 7793 have morphologies and photometric properties similar to Milky Way GCs. Assuming a contamination rate of $\sim 50 \%$, the sample sizes imply specific frequencies of at least $S_{N} \sim 0.2-0.5$, as is typical for late-type galaxies. With spectroscopic confirmation of the GC candidates, we expect to have sufficient numbers of GCs to study their global kinematics.

\section{References}

Beasley \& Sharples 2000, MNRAS, 311, 673

Bertin \& Arnouts 1996, A\&AS, 117, 393

Blecha 1986, A\&A, 154, 321

Chandar 2002, IAU Symposium 207, "Extragalactic Star Clusters"

Da Costa \& Graham 1982, ApJ, 261, 70

Johnson et al. 1999, ApJ, 527, 199

Liller \& Alcaino 1983, ApJ, 264, L53

Liller \& Alcaino 1983, ApJ, 265, L166

Mighell et al. 1996, AJ, 111, 2314

Miller et al. 1998, ApJ, 508, L133

Olsen et al. 1998, MNRAS, 300, 665

Olszewski et al. 1991, AJ, 101, 515

Sarajedini et al. 1998, ApJ, 508, L57

Schommer et al. 1991, AJ, 101, 873

Schommer et al. 1992, AJ, 103, 447 

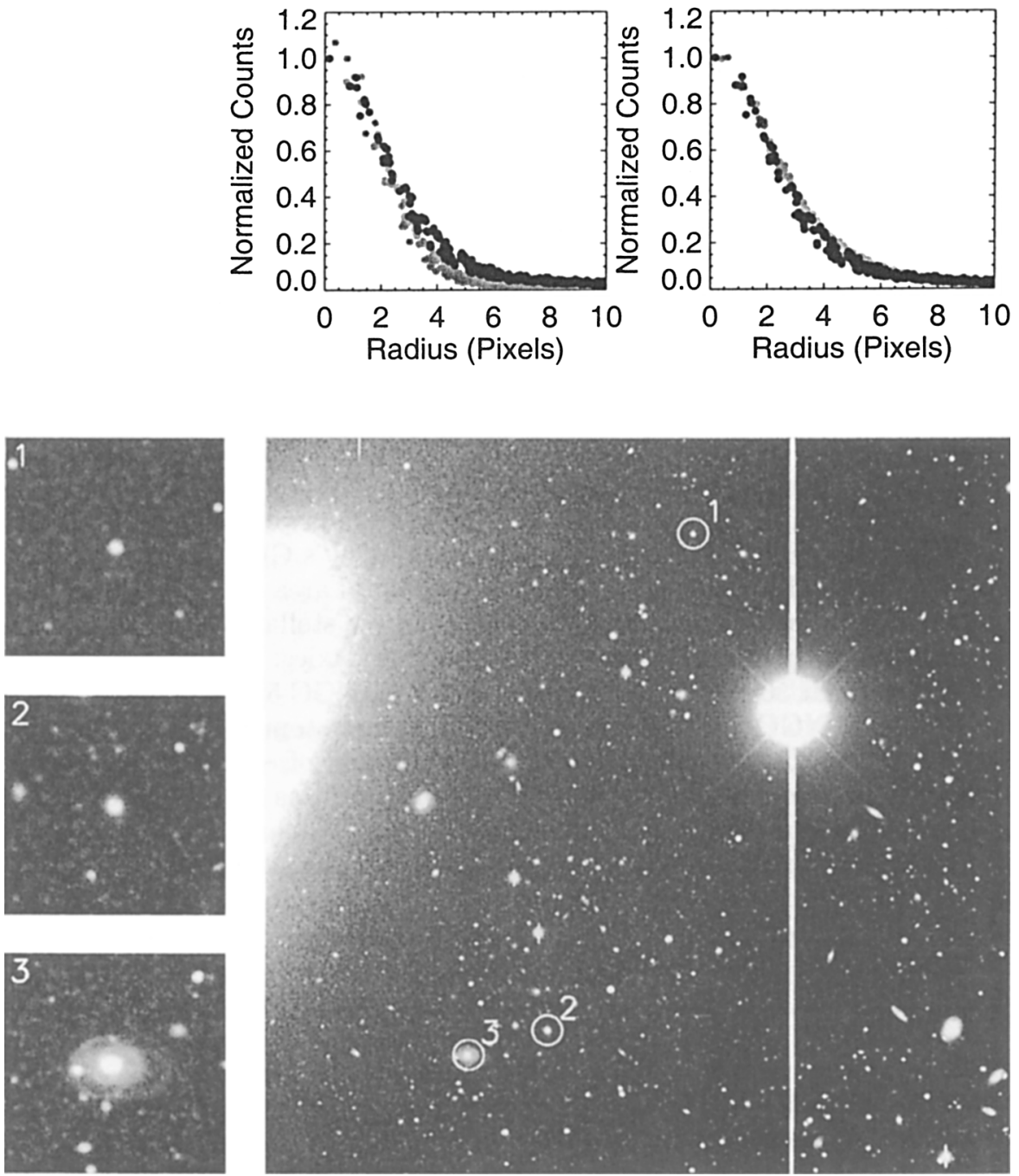

Figure 1. A $2 \mathrm{~K} \times 2 \mathrm{~K}$ section of the full $8 \mathrm{~K} \times 8 \mathrm{~K}$ Mosaic- $2 R$ image of NGC 253, featuring three objects which appear as globular cluster candidates on photographic plates. The objects are displayed in closeup in the images on the left. Spectroscopy has shown (Beasley \& Sharples 2000) that object 1 is a Sculptor Group globular cluster, while objects 2 and 3 are background galaxies $\left(v \sim 23000\right.$ and $33000 \mathrm{~km} \mathrm{~s}^{-1}$, respectively). In our image, object 3 is clearly a spiral galaxy, obviating the need for spectroscopy to confirm the nature of this object. Our image is unable to identify object 2 as a galaxy in the same fashion. The radial profile shown at top left compares the profile of the globular cluster, object 1 (black points), with that of a typical star (grey points); that at top right plots the profiles of object 1 (black points) and of the galaxy, object 2 (grey points). Both objects 1 and 2, but not object 3 , would be selected as globular cluster candidates through our analysis. 

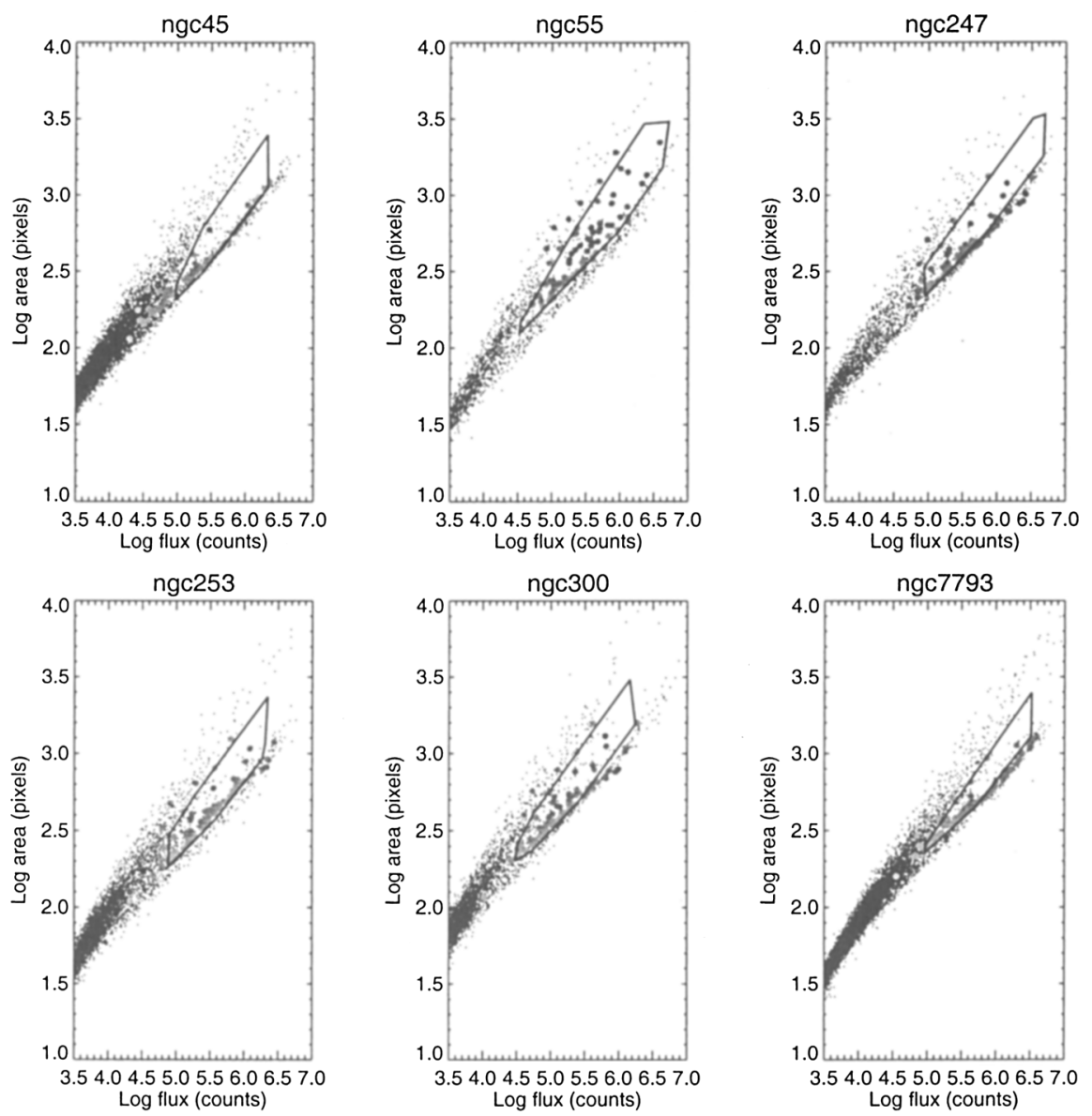

Figure 2. The area within the isophote having surface brightness $3 \sigma$ above the sky background is plotted vs. the $R$-band integrated flux within the isophote, as measured for objects found in deep Mosaic II images of six Sculptor Group galaxies (small points). Only those objects with $e<0.4,0.25<M-R<0.8$, and $0<C-R<2.4$ are shown; objects projected against the crowded galaxy disks have also been masked out. The gray circles show where the Milky Way's globular clusters would appear as seen at the distances of the Sculptor Group galaxies. The boxes indicate the areas in which we concentrate our search for Sculptor Group globular cluster candidates. Each box contains $\$ 100$ candidate clusters with properties similar to the Milky Way globular clusters. We expect that $50 \%$ of the candidates are true clusters, as judged by the equal number of spectroscopically confirmed clusters and galaxies (Beasley \& Sharples 2000) found in the NGC 253 box. 


\section{Discussion}

C. Grillmair: Your cluster selection criteria seem to have missed a number of convolved Milky Way clusters.

$K$. Olsen: Our criteria were optimized to reduce the contaminating number of foreground stars. 